\title{
Flood risk management with HEC-WAT and the FRA compute option
}

\author{
Christopher Dunn ${ }^{1, a}$, Penni Baker ${ }^{2}$ and Matthew Fleming ${ }^{3}$ \\ ${ }^{1}$ Director - U.S. Army Corps of Engineers, Institute for Water Resources, Hydrologic Engineering (CEIWR-HEC), 609 Second \\ Street, Davis, CA 95616, United States of America \\ ${ }^{2}$ Software Programmer, U.S. Army Corps of Engineers, Institute for Water Resources, Hydrologic Engineering Center \\ (CEIWR-HEC), 609 Second Street, Davis, CA 95616, United States of America \\ ${ }^{3}$ Senior Hydraulic Engineer, U.S. Army Corps of Engineers, Institute for Water Resources, Hydrologic Engineering Center \\ (CEIWR-HEC), 609 Second Street, Davis, CA 95616, United States of America
}

\begin{abstract}
United States Army Corps of Engineers (USACE) policy states that USACE will analyze water resources projects, including flood risk management projects, using a risk framework that incorporates watershed, systems and life-cycle approaches. However, software to directly support these requirements has not been available. The current software used within USACE to perform these types of analyses, HEC-FDA (Flood Damage Reduction Analysis), has a number of limitations. For this reason and others, USACE's Hydrologic Engineering Center (CEIWR-HEC) developed the Watershed Analysis Tool (HEC-WAT). HECWAT was initially developed to assist USACE personnel conduct water resources studies of complex riverine systems with an integrated, comprehensive and systems based approach. However, since USACE also requires a life-cycle analysis, CEIWR-HEC added capability through the Flood Risk Analysis (FRA) compute option that allows risk analysis computations while incorporating a life-cycle approach. HEC-WAT/FRA includes systems and life-cycle approaches, event-based sampling, parameter sampling, and the ability to do scenario and alternative analyses. Applications of the FRA compute include levee certification studies, dam and levee safety studies, and planning and design studies. This paper will introduce HEC-WAT/FRA, describe scientific advancements included within it and provide applications to demonstrate how it will advance USACE modeling approaches.
\end{abstract}

\section{Introduction}

One of the primary missions of the United States Army Corps of Engineers (USACE) is to manage the nation's water resources. For a variety of reasons, including budgetary, it is critical that the nation's water resources be studied and managed using a collaborative and comprehensive approach implementing system and watershed concepts while incorporating risk and life-cycle analyses.

To support these requirements, USACE has written several pieces of guidance and policy that state that water resource studies shall be performed with risk analysis techniques using an integrated [1] [2], comprehensive, and systems based approach. However, implementing true watershed and systems approaches with risk and life-cycle analysis can be difficult. USACE currently certified flood risk management tool, HEC-FDA (Flood Damage Reduction Analysis), represents the system as a number of independent projects rather than an integrated system [3]. This limitation, along with a few others, was identified by the National Research Council, National Academy of Sciences in their 2000
Report, Risk Analysis and Uncertainty in Flood Damage Reduction Studies [4]. Therefore, a new water resources planning tool needed to be developed that would include explicit systems, watershed and life-cycle capabilities along with the risk analysis computations currently found in HEC-FDA.

To address these issues USACE has developed HEC-WAT (Watershed Analysis Tool) to study and manage the nation's water resources in a systems and watershed approach while incorporating risk and lifecycle capabilities [5]. The HEC-WAT framework integrates the software commonly used by multidisciplinary teams to perform comprehensive system type studies. HEC-WAT also allows a Project Delivery Team (PDT) to perform alternative analyses in an intuitive and collaborative manner.

However, while the software addresses many of the requirements and recommendations that provoked its development, certain aspects of the development are still a work in progress. A number of technical challenges need to be overcome before USACE can claim it has a truly universal life-cycle, systems and watershed tool. These challenges and solutions to some of these challenges are discussed.

\footnotetext{
a Corresponding author: Christopher.n.dunn@usace.army.mil
} 
In spite of the fact that some capabilities are not finalized, a number of USACE offices have found value in the tool and have begun to use HEC-WAT to solve a variety of problems thus demonstrating the versatility of the tool. Applications include large studies on the Columbia, Missouri, and Russian Rivers, Red River of the North, and a number of dam and levee safety evaluations.

In addition to the applications in the United States, interest in HEC-WAT is becoming global. The need for tools that can perform systems based and watershed approaches while addressing risk and uncertainty is not unique to the United States. Presentations about the capabilities of HEC-WAT have been made in Brazil [6] and South Africa [7] and the software has been requested by a number of Countries (Spain, Afghanistan, Netherlands) outside of the United States.

\section{HEC-WAT}

HEC-WAT is an interface that streamlines and integrates a water resources study using software commonly applied by multi-disciplinary teams that comprise a typical PDT. Many pieces within the CEIWR-HEC suite of software are implemented within HEC-WAT thus allowing a study team to perform many of the necessary hydrologic, hydraulic, and planning/consequence analyses from a single interface.

HEC-WAT also allows the PDT to perform an alternative analysis in an intuitive and collaborative manner. This ease of use provided by the HEC-WAT framework improves a study team's ability to facilitate, convene, advise, and work collaboratively. HEC-WAT strives to involve modelers early in the study process, which encourages a collaborative team approach.

HEC-WAT is not replacing existing software but rather the framework allows unique pieces of software to work together. The model integration of the individual pieces of software within the HECWAT framework is achieved through the concept of a "plug-in". The plug-in is what allows the individual pieces of software to integrate without requiring special code in HEC-WAT to support the individual pieces of software (Figure 1). There are numerous ways to implement plug-ins; therefore, the plug-in concept should allow a plug-in to be developed for any software that might be included in the HEC-WAT framework. With the plug-ins, HEC-WAT provides the analysis framework, but does not know anything about the individual pieces of software. The individual pieces of software provide the editors, reports, computational analyses, etc. and are not intended to contain HEC-WAT specific code. HECWAT provides a framework to coordinate the study, while the individual pieces of software provide the analytical computations. As the HEC-WAT matures, additional pieces of software outside of the CEIWRHEC family can be incorporated.

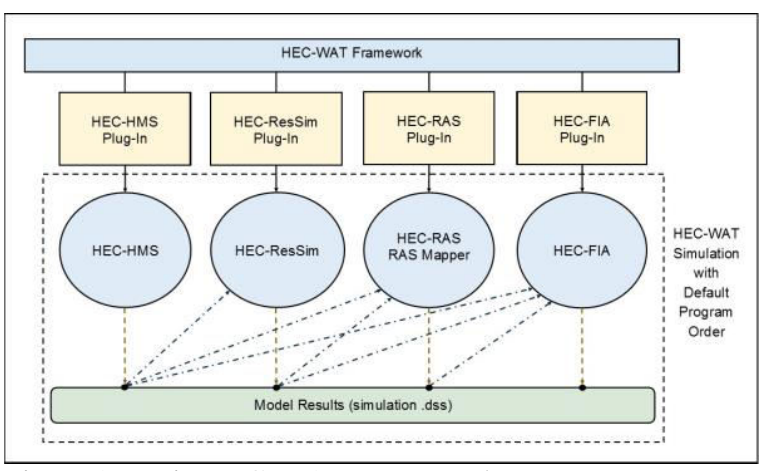

Figure 1 Basic HEC-WAT Framework

The major pieces of software currently implemented in HEC-WAT are shown in Figure 1.

- HEC-HMS (Hydrologic Modeling system) is a standalone application designed to simulate the precipitation-runoff process of a watershed system. In the HEC-WAT sequence, HEC-HMS is a hydrologic rainfall-runoff model that provides flow data into and downstream of reservoirs.

- HEC-ResSim (Reservoir System Simulation) is a standalone application that is used to model reservoir operations at one or more reservoirs whose rule-based operations are defined by a variety of operational goals and constraints. The software simulates reservoir operations for flood management, low flow augmentation and water supply for planning studies, detailed reservoir regulation plan investigations, and real-time decision support. In the HEC-WAT sequence, HEC-ResSim provides peak flow/stages, flow hydrographs, or stage hydrographs to downstream control points and the hydraulics model.

- HEC-RAS (River Analysis System) is a standalone application that allows the user to perform one- and two-dimensional flow, sediment transport/mobile bed computations, and water quality and water temperature modeling. The HEC-RAS Mapper tool available from the HEC-RAS interface calculates inundation boundary and water depth maps. HEC-RAS computes the river stages, water surface profiles, and levee breach information that is used to compute consequences via HEC-FIA in the HECWAT sequence.

- HEC-FIA (Flood Impact Analysis) is a standalone application that evaluates consequences using either observed or forecasted hydrographs (hydrograph-based) or depth grids (Geographic Information System (GIS) based). For a specified analysis, the software evaluates urban and agricultural flood damage, area inundated, number of structures inundated, and consequences. The consequences include economic and life loss. HEC-FIA also provides information on actions for emergency operators.

The HEC-WAT tool allows USACE and its partners and stakeholders to conduct their studies in a coordinated fashion. Coordination begins as each model uses the common schematic that is built within the HEC-WAT interface. The common schematic 
demands that each team use the same nomenclature for each of their models and alternatives. A schematic (Figure 2), is usually built from background layers such as shapefiles and DEM's (digital elevation model) to visually represent the

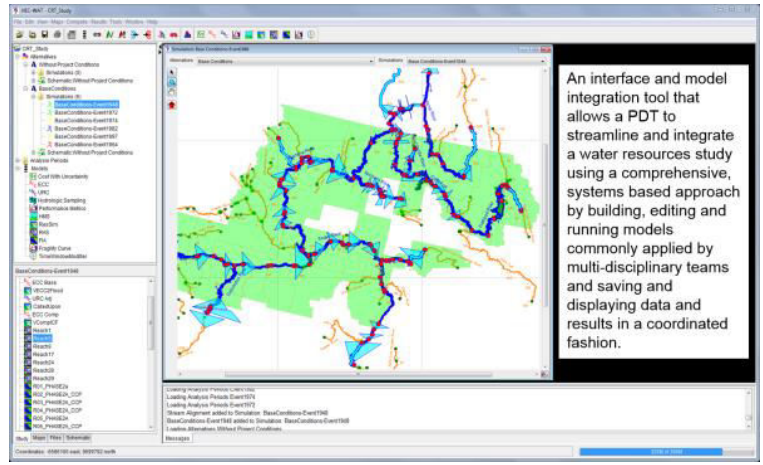

Figure 2 HEC-WAT Schematic - Columbia River System

watershed and to provide a spatially correct representation of the watershed. The individual models can be built and edited in or outside the HECWAT and model results are viewed by selecting the elements found on the schematic. Once the models are in HEC-WAT, the models can be run in sequence. The storage of data is organized by DSS (Data Storage System) and a simple DSS linking device is used to connect the models. The models themselves, the input data, and the results are all stored in the HEC-WAT's directory structure. Therefore, all data and files used to make decisions are easily retrieved. Alternative analyses can be performed and output from multiple alternatives can be viewed at the same time making alternative analyses and selection easier.

The basic building block of a schematic is the stream alignment. The stream alignment is a representation of the streams as they travel through the watershed. Because all models will share the naming convention and geo-referencing data, the stream alignment will generate a consistency between models and study alternatives.

The next important schematic element is the common computation point (CCP), which is a location where a model transfers data to other model. CCPs could also be locations where results are needed for model development or alternative analysis. The CCPs will be consistent for all models and should be placed on the schematic based on knowledge of possible study alternatives. Other elements that need to be defined in the shared schematic are flood damage reduction and/or ecosystem restoration measures. All objects within the schematic, the watershed area, stream alignment, CCPs and flood damage reduction or ecosystem restoration measures can be used across multiple alternatives.

The shared schematic and models that use the schematic are defined as an alternative. With the addition of events and time periods to be used by all the models, simulations are created which facilitate system analysis. From the HEC-WAT interface the user can edit a model and then run one model at a time or re-run the entire simulation. For example, once the models are built, the modeling team may want to see if a different infiltration rate would lead to higher flows and thus increased damage.

Using consistent schematics, data, and tools, alternative results will be easier to compare making the trade-off analysis and selection easier as well. Both ecosystem restoration and flood risk management alternatives will eventually be created directly within HEC-WAT. To view any hydrologic element (computation points, reservoirs, river reaches, impact areas, storage areas etc.), the element can be selected on the schematic and a list of tabular or graphical output will be available. The user could be oblivious to which model actually produced the results.

\section{HEC-WAT, Flood Risk Analysis Option}

For over two decades, USACE has required all USACE planning processes use risk analysis and lifecycle approaches. However, as previously discussed, there are few tools to support these requirements. Therefore, CEIWR-HEC created a compute option within HEC-WAT, called Flood Risk Analysis (FRA) that helps analyze complex riverine systems while addressing flood risk management, system and lifecycle requirements (Figure 3 ).

The FRA option includes sampling and solution techniques, uncertainty definitions, and system-wide component fragility and performance interactions/relationships for these complex riverine systems. The capabilities evolved from previous efforts and are detailed in the software design document [8]. HEC-WAT can now be used nationwide for levee certification, levee assessment, planning and design studies and advance USACE's modeling approach for risk and life-cycle analysis.

The FRA computational methodology starts with the definition of a project alternative and a single scenario of the project life cycle (e.g., fifty years) being simulated by sampling the flood events over the duration of the life cycle. The system performance, that is, how the levees perform during an event, and flood characteristics will determine when and if breaches occur. If breaches do occur, the impacts will be calculated based on flood inundation and related consequences. The consequences shall include economic, environmental and life loss. Life cycle modeling will include the deterioration of projects over time, rehabilitation, repair and flood recovery when necessary. Using the life cycle approach accounts for situations when damage is substantial and the characteristics of the consequence area are altered prior to the next flood event, thus not double accounting for flood losses.

The FRA option performs a Monte Carlo analysis, during which sampling of not only flood events but uncertainties about hydrologic, hydraulic, reservoir operations, geo-technical and economic/consequence parameters occur. When an 


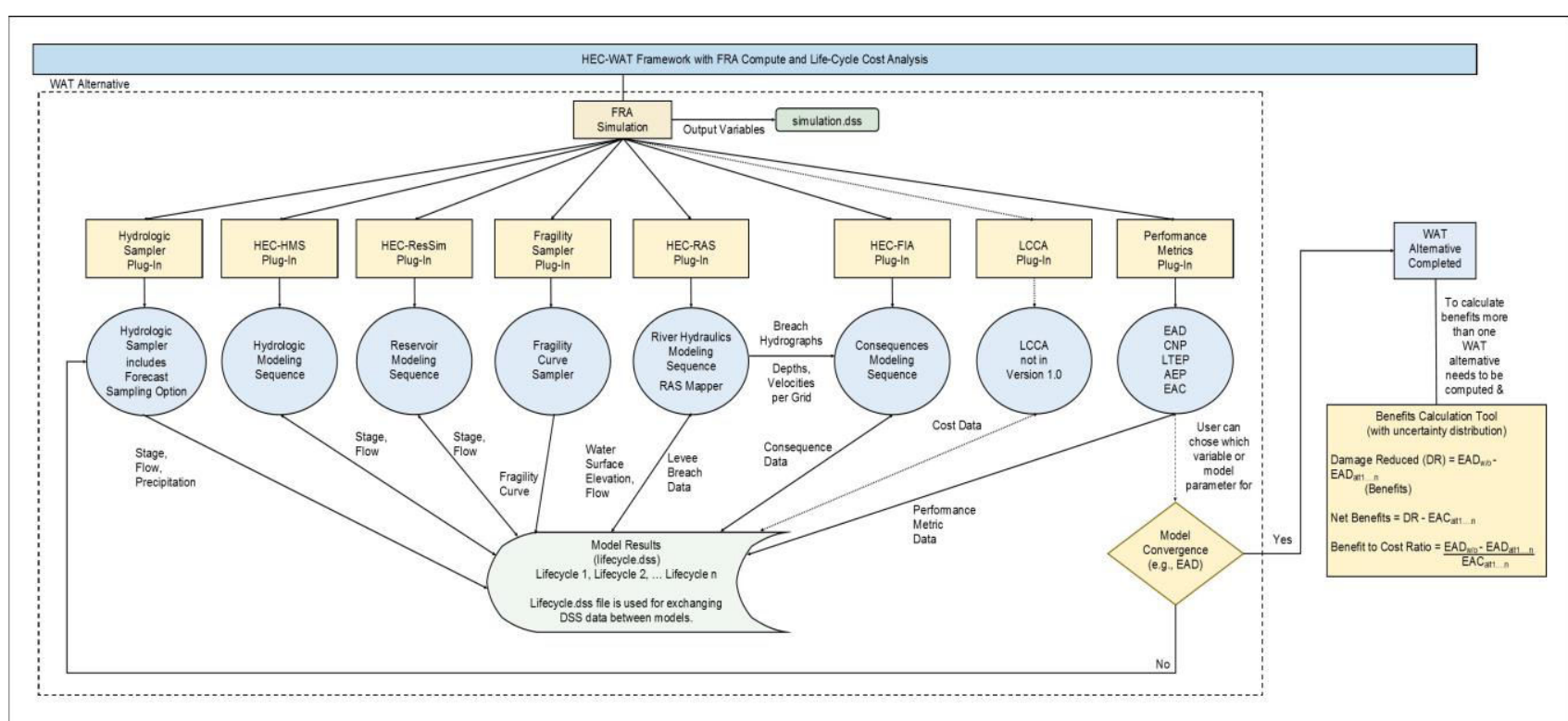

Figure 3 HEC-WAT Framework with FRA Compute Option

FRA compute is initiated, the model alternatives for that particular run are executed repeatedly for sampled flood events and model parameter estimates until convergence about the EAD (Expected Annual Damage) is achieved. Computational methodologies had to be created, since the FRA compute will be quite intensive. For large studies, distributed computing and use of multiple processors may be required. The following paragraphs describe a few of the new methodologies that have been implemented within the FRA compute option. A more thorough description of each is provided within other papers written for previous conferences.

The sampling of hydrology to create thousands of years of annual flood flows is the first step and occurs in the Hydrologic Sampler. The realizations of the hydrologic record will be divided into fifty-year life-cycles. With FRA performing an event-based analysis, the hydrology information is needed as a time series of flow to be used by other models in HEC-WAT to then be routed through the system under study.

To capture the likelihood of levee failure, fragility curves will be sampled at each project location for each event. In a systems context with multiple failure modes/locations, sampling of those fragility curves, including uncertainty, is required for each realization of an FRA simulation. If the computed water surface elevation meets or exceeds the stage sampled from the fragility curve, the levee is assumed to fail and water spills into the interior area. After a large number of simulations, this would result in the evaluation of the full range of failure possibilities over the entire system. This sampling and failure routine would define the system load distributions as well as inundation delineations in the consequence areas of interest.

In order to evaluate and compare alternative flood risk measures, including levees, USACE utilizes several economic and performance metrics. These metrics are used to assist in communicating risk as required in USACE guidance [9]. These include EAD, Annual Exceedance Probability (AEP), Conditional Non-exceedance Probability (CNP) or assurance and Long-Term Exceedance Probability (LTEP). HECWAT with the FRA compute option computes each of these metrics.

EAD is the mean or average of all possible values of damage. In FRA, computing EAD and the distribution of the EAD starts by sampling the flow frequency curve and then ultimately compiling damage for 500-year realizations. As EAD is computed for each 500 year period, these 500-year estimates of EAD will be stored as a population of EAD estimates. Once the final mean EAD is computed, then the population of EAD estimates can be used to define a distribution of EAD.

AEP is the probability of getting wet at a given location (i.e., consequence area location, grid cell, threshold stage location, or a fragility curve location) in any given year considering the full range of possible floods and project performance. During a Monte Carlo simulation, the AEP is estimated based on how many times an area was recorded as being wet or a stage exceeded versus the total number of events. Like the EAD estimates, an estimate of AEP will be generated for each realization, and a resulting sample of AEP estimates will provide the AEP distribution.

CNP or "assurance" is the probability that a project will be able to contain a flood of specified frequency. For example, USACE requires that, for a levee system to be found in accordance with National Flood Insurance Program (NFIP) levee system evaluation requirements, it must have at least a ninety percent chance of containing the 1 percent annual chance exceedance flood. In an FRA compute, assurance will be computed at predefined locations within the system, generally defined by the location of system components and their associated fragility functions. 
LTEP is the probability that a target stage or system component will be exceeded at least once during the course of a specified term (e.g., thirty years, the typical life of a home mortgage).

\section{Challenges}

While the software addresses many USACE requirements and also meets many of the recommendations lined out by the National Academy of Sciences, National Research Council, it is still a work in progress. A number of technical challenges still need to be overcome. Some of them include: incorporating life-cycle modeling that addresses rehabilitation, repair and flood recovery; calculating expected annual costs and then combining them with expected annual damage; performing a consequence evaluation across economic, social, environmental and life loss and then making a decision across these different metrics; using uncertainty analysis trade-offs between detailed modeling and important sources of uncertainty; communicating risk; incorporating and modeling changes to the climate and watershed; modeling multiple failure modes; and addressing planning transformation and the future of planning analysis in USACE.

Additional challenges include moving CEIWRHEC software into the future by making the software more intuitive, faster, more automated, improving graphics capabilities, and providing software that is stable, robust, and familiar. USACE needs to continuously improve the software to address these expectations plus continue to develop software that meets the changing needs of USACE, the country and the profession.

Of the technical aspects of the HEC-WAT modeling approach, reduction of the computational burden is the largest challenge. To reduce the time required to make large runs over many years and many realizations, CEIWR-HEC has developed or made use of a few approaches including Distributed computing, the Model Skip Rules Tool and the Time Window Modification Alternative. These are discussed below.

\subsection{Distributed Computing}

To adequately perform a risk analysis for an entire watershed using a systems approach and accounting for uncertainties associated with both knowledge uncertainty and natural variability across a series of disciplines, many parameters must be sampled across most if not all events and for most if not all models. In addition, to explicitly address a systems approach within a large watershed, all components of the watershed must be somewhat interrelated so upstream actions are reflected in downstream impacts as well. For example, if a levee breached upstream, the volume of water lost due to the upstream breach must be accounted for as the flood travels downstream. Therefore, one model must inform the next. To accommodate these requirements, the sampling of hundreds of thousands if not millions of events is required for the evaluation of large, sophisticated projects. The sampling of these parameters and the running of these models then requires an enormous amount of compute time. The long compute times are a logical outcome of satisfying the USACE requirements of using watershed and systems approaches for planning projects while also employing risk assessment and life-cycle concepts. In large watersheds with numerous models approximating numerous physical processes, the overall simulation for only one event can take hours on a single computer. Even a simulation time of a couple of minutes multiplied by many thousands of events can lead to extremely long simulation times. Therefore to reduce the compute time, CEIWR-HEC is developing the ability to perform distributed computing in HEC-WAT. For example, to run a simulation of a complex system on one machine, the entire simulation would take tens of thousands of hours. Neither the project team nor the customer can wait that long for a single compute. The distributed computing capability greatly reduces the time it takes to perform a single simulation for one alternative in a complex system from months to a few hours. The distributed computing option is scalable; it can be performed from a single computer with multiple virtual machines, a Local Area Network of several computers, or on a cloud service. Ongoing work to improve a distributed compute includes adding capabilities to monitor the status of the simulation and report when individual compute nodes have finished. This feature allows others to track the progress of the simulation and to quickly shut down unnecessary computing resources. In addition, measures are being implemented to keep the simulation running when model, software, or hardware failures are experienced during the simulation. A model going unstable, a temporary lost connection, or the complete failure of a compute node should not cause the simulation to stop. Robust measures that keep the simulation running and note events to be evaluated are necessary when running simulations that take multiple days to complete.

\subsection{Time Window Modification}

The Time Window Modification Alternative provides capabilities for dynamically modifying the time window used by each model in the compute sequence so that models do not have to share the same simulation time window. An added benefit of the Time Window Modification Alternative is the ability to find a starting time that meets boundary conditions and at the same time does not cause models to go unstable due to initial boundary conditions.

The simulation time window is useful when individual models, like HEC-ResSim or HEC-RAS, are called to run in a HEC-WAT/FRA compute. One of the features built into the FRA compute is the 
ability to modify the time window used by each model in the compute sequence. This capability can be a time-saving feature due to the fact that the HECResSim models are often required to run a simulation time-window that spans the entire water year in order to evaluate reservoir operations. HEC-RAS and HEC-FIA models, meanwhile, do not need a simulation time window for the entire water year. Their required output is consequences or damage from a certain time period such as a snowmelt season from April to June or from the largest flood event in the year. Two methods are available for modifying the time window in the FRA compute, one is to edit the time window defined in the simulation editor, and the other is to create a Time Window Modification Alternative. The Time Window Modification Alternative is loosely treated as a model because it performs an analysis (evaluates time-series data) and generates output (returns a modified time window).

The Time Window Modification Alternative contains additional flexibility for modifying the time window. In order to use, a Time Window Modification Alternative is created, and must be included in the HEC-WAT/FRA compute sequence. A dataset must be associated with each Time Window Modification Alternative. This association to a dataset is accomplished within the HEC-WAT model using the Model Data Linking Editor.

Figure 4 shows the Time Window Modifier Alternative Editor, which for example is setup to define the simulation time window for a reach in an HEC-RAS model. The Time Window Modifier

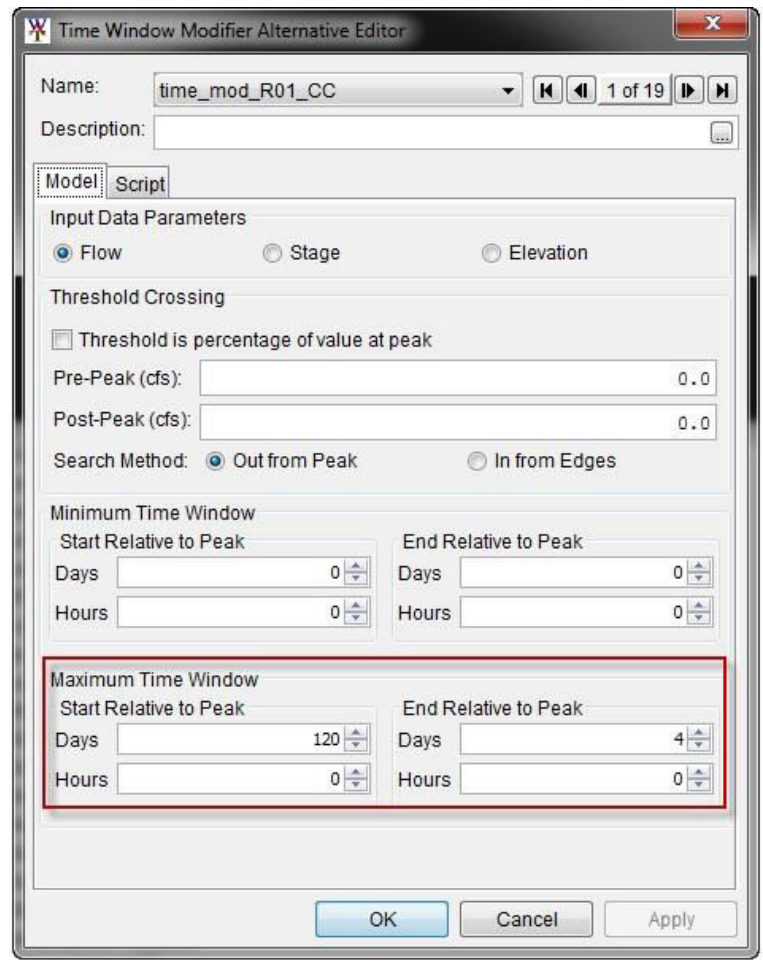

Figure 4 Time Window Modifier Alternative Editor

Alternative Editor contains three options for modifying the time window which centers around the peak flow/stage from the time-series assigned to the alternative. One option includes user-defined thresholds (defined numerically or as percentages of the peak value) before and after the peak flow/stage. Another option is to set the minimum time window centered on the peak flow/stage. The final option is to set the maximum time window centered on the peak flow/stage. The program will move forward and backward in time from the peak flow/stage until the user-defined thresholds or number of days/hours criteria is met.

Model instability due to initial conditions is an important issue to address for Monte Carlo simulations. Initial conditions outside the range experienced when initially calibrating models to observed data are likely in Monte Carlo simulations that include thousands of events. For example, initial simulations of the Columbia River Treaty (CRT) Review FRA compute contained a number of instances of model failures due to initial conditions. A solution was developed to enhance the Time Window Modifier to evaluate multiple datasets (Figure 5), not just one, while using a scripting environment to allow customization of the logic used to modify the simulation time window. The scripting feature is used in conjunction with the other options

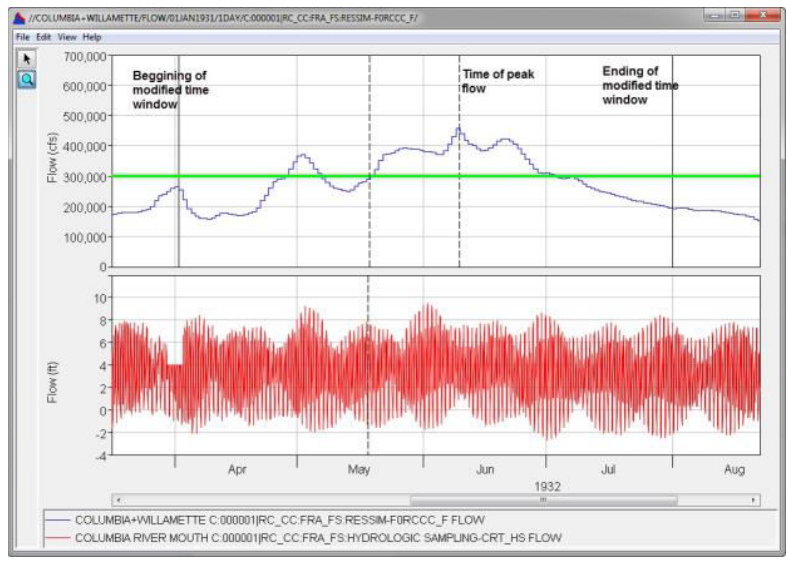

Figure 5 Plot of Two Boundary Conditions Time-Series for an HEC-RAS Model

already contained in the Time Window Modifier Alternative Editor. The goal of the new scripting feature applied to the CRT model was to find a new starting time where the boundary conditions, timeseries computed by other models or randomly sampled, did not cause the HEC-RAS models to go unstable. This shortened time window can save minutes of compute time for each run but if multiplied by thousands of events this reduction is a considerable improvement.

\subsection{Model Skip Rules}

The Model Skip Rules tool provides an interface for defining thresholds (flow, stage, or other model output) that are used to determine when certain models in the compute sequence are skipped. For example, a study evaluating damages due to high flows can use the Model Skip Rules tool to skip all 
events where the sampled flow magnitude is lower than the flow threshold that results in damage. Neither the hydraulics nor consequence models would need to be run for the skipped events, saving a large amount of simulation time.

Not all events sampled during an HEC-WAT FRA simulation will result in consequences; the computed flows and water surface elevations will not be large or high enough to generate damages. In these cases, it might not be necessary to simulate the event. The Model Skip Rules toll, will allow the user to build logic expressions for evaluating whether specific models in the compute sequence are simulated for an event in the FRA compute.

For example, the model skip rules were used extensively in the CRT model simulations. The reservoir modeling sequence (made up of multiple reservoir models) was computed for all events in the FRA compute in order to evaluate reservoir operations; however, the HEC-RAS and HEC-FIA models were only simulated when flows from the reservoir models exceeded user-defined thresholds. These threshold flows were developed by the CRT modeling team and represent flows where no damages would be expected.

The time savings from the Model Skip Rules Tool can be considerable. Based on the conservative skip thresholds, approximately thirty-five percent of the events can be skipped. A higher percentage of events can be skipped where damages are not realized until large, rare flood events are simulated.

\section{Applications}

\subsection{Columbia River Treaty (CRT) Review}

The Northwestern Division (CENWD) of USACE, along with several stakeholders needed to perform a series of hydraulic and hydrologic technical studies to collect critical information to support a pending decision by the United States pertaining to the future of the Columbia River Treaty with Canada. A requirement of the review was to develop a comprehensive, systems evaluation approach for the Columbia River Basin and to evaluate the current and future flood risk within the Basin (Figure 6) which included hydropower operations. Because of these requirements the CENWD PDT determined that the HEC-WAT/FRA was the tool needed to evaluate treaty alternatives using EAD and other criteria.

Models implemented within HEC-WAT were developed by the CRT PDT to help understand the level of flood risk from the current operation of the system and to evaluate the flood risk from alternative reservoir operation scenarios (both in Canada and the United States). Sixty-five reservoirs were modeled using multiple pieces of software. These models also included CENWD local software, URC (Upper Rule Curves) and ECC (Variable Energy Content Curves). The HEC-ResSim software was used to create a

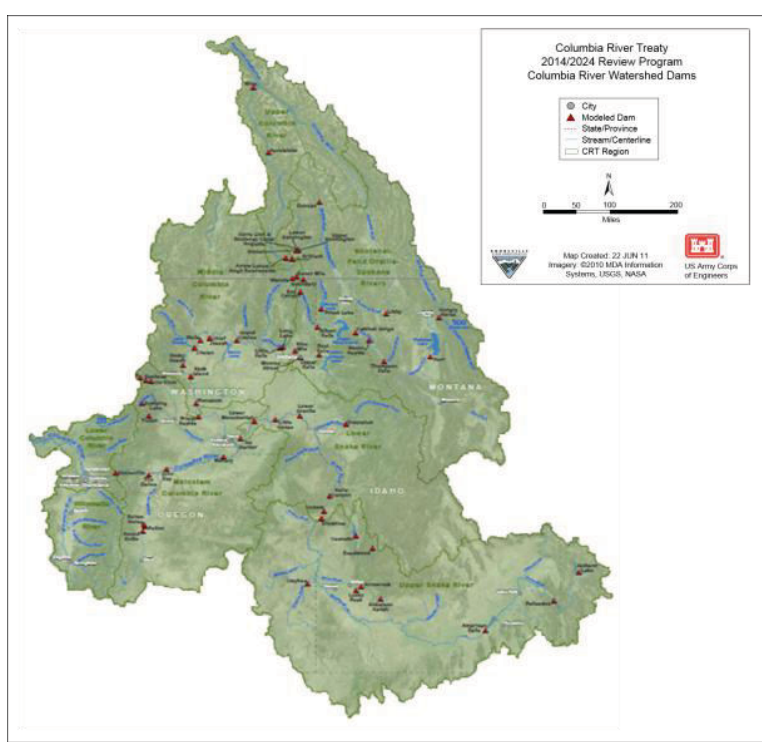

Figure 6 Columbia River Basin

model for hydropower operations and a model for flood control.

Twenty-six hydraulic models were developed along major river segments using the HEC-RAS software. Eight unsteady HEC-RAS models were included directly in the FRA compute sequence. The other eighteen steady flow models were used to develop flow-stage relationships that were provided to the reservoir software in order to compute a stage time-series from the simulated flow. For consequence modeling, the HEC-FIA software was used for the twenty-six identified river segments. The CRT HECFIA model included a structure inventory of over 185,000 structures.

Seventy years of hydrologic data (flows and forecasts) were collected and assembled including sixteen synthetic floods that were also generated. Fragility curve information for the levee systems within the basin were also collected and entered into the hydraulic models. The fragility curves were used to sample levee failure elevations, which were used by the unsteady flow HEC-RAS models.

Deterministic runs of the HEC-WAT CRT watershed were performed to create a baseline for verification of the FRA compute results. The deterministic runs included the reservoir modeling only and then runs that included all the models. The FRA computes started with 5,000 event simulations of the reservoir modeling only (average 1.75 days for one simulation using eleven computers); there were about seventy of these simulations run.

Once the reservoir modeling results were verified by the CRT PDT, the FRA computes were conducted on the full sequence of models. FRA computes of 5,000 events were performed for several alternatives, each averaging about fourteen days using ten computers. For the 50,000 event FRA computes, the average execution took fourteen days but using 100 computers through cloud computing. For these, the execution time was an average of about twentyfive minutes per event with distributed computing and 
other time reducing capabilities (e.g., narrowing of time windows for specific models, skipping of models based on certain thresholds, improvements to various applications being used in the computing sequence) being employed. At the beginning of the process, computes were averaging about ninety minutes per event.

By the end of the CRT Study, the U.S. Entity had sufficient information from the HEC-WAT/FRA study results and from other sources to write a position paper on the future of the CRT after 2024 [10]. They recommended the Treaty be modernized to reflect the current and future needs of the Columbia Basin. This decision may not have been as defensible without the development and implementation of HEC-WAT/FRA.

\subsection{Missouri River Study}

CEIWR-HEC is providing technical support to the Omaha, Nebraska and Kansas City USACE offices on the development of HEC-ResSim and HEC-RAS models for the Missouri River Basin (MRB) as part of the Missouri River Recovery Program (MRRP). The MRRP is an umbrella program for coordinating activities on the Missouri River for restoration of native habitats and to comply with the Endangered Species Act and the 2003 Biological Opinion. The model development is part of a hydrologic and hydraulic analysis to serve the MRRP study of long term ecological and socio-economic benefits, and also to demonstrate compliance with National Environmental Protection Agency (NEPA) regulations. Model development is in the preliminary stages, with a target of calibrating the standalone HEC-ResSim and HEC-RAS models. Once the models are finished, an HEC-WAT watershed, patterned after the CRT example will be created to simulate and analyze a variety of study alternatives. However, in this case, the HEC-WAT project could also include CEIWR-HECs Ecosystem Functions Model (HEC-EFM) to assist with the evaluation of the ecosystem impacts along with the hydraulic and reservoir results. HEC-EFM is a planning tool that uses hydrologic and hydraulic data to help predict biological response to flow regime changes. The hydrology and hydraulics data works as surrogates to environmental data that is often difficult to find. The questions posed for this study could not just be answered by hydrology and hydraulics data alone.

\subsection{Russian River Study}

CEIWR-HEC is performing an analysis using HEC-WAT that evaluates the use of Forecasted Informed Reservoir Operations (FIRO) for the possible reoperation of the Coyote Valley Dam (Lake Mendocino) which is located in the Russian River Watershed. The Russian River watershed is approximately 1480 square miles $\left(3833 \mathrm{~km}^{2}\right)$ and is located in northern California, see Figure 7. The

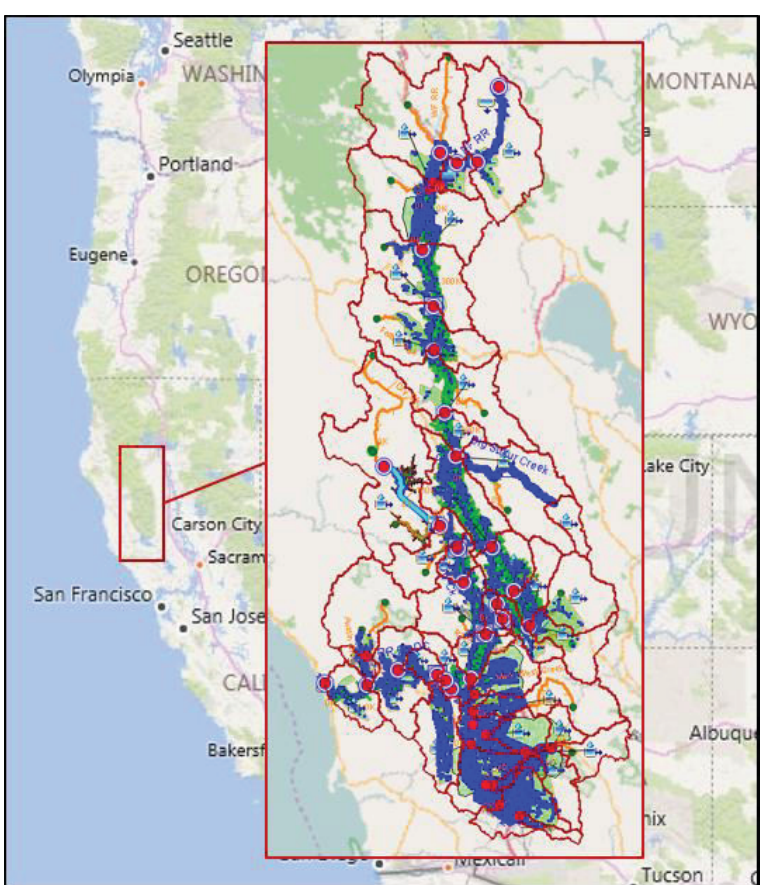

Figure 7 HEC-WAT Russian River Study

purpose of the study is to determine if increases in water supply could be gained while maintaining the current flood and environmental missions. USACE operates two reservoirs in the watershed, Coyote Valley Dam and Dry Creek Dam, however, the study will initially focus on the Coyote Valley Dam. Historically, inter-basin flows from the Eel River watershed have contributed to the water supply in the upper Russian River watershed as the transferred water was stored in Coyote Valley Dam. However, the Eel River contribution has been cut drastically and there is a need to update reservoir operations for Coyote Valley Dam to provide a more reliable water supply, especially given the cycle of drought and flood experienced in the Western United States. The premise behind FIRO is that the ability to predict significant floods events is improving and, therefore, maintain higher reservoir pool elevations in the flood season to hold onto water longer and only release water if larger events are forecasted. This study will evaluate whether flood risk and environmental considerations could be maintained when incorporating forecasted precipitation and soil moisture into reservoir operations for the Coyote Valley Dam. Lessons learned from the evaluation of the Coyote Valley Dam could be transferred to the Dry Creek Dam for evaluating reservoir operations and their impact on the entire Russian River watershed. For this implementation of HEC-WAT, HEC-HMS, HEC-ResSim, HEC-RAS and HEC-FIA models will be run for a Period-of-Record and then an FRA compute.

\subsection{Red River of the North}

HEC-WAT is being used in several climate studies being conducted within USACE. The Red 
River of the North Climate study used HEC-WAT to link HEC-HMS and HEC-ResSim models that simulate precipitation, runoff, and reservoir operations in the Red River watershed. Precipitation and temperature model output from global climate models was used as boundary conditions for the HECHMS model. Since the global climate models generate data at large spatial and temporal scales (one-month time step), a statistical downscaling method was used. This method takes the monthly output from the global climate models and uses historical temperature and precipitation patterns to develop multiple possibilities (randomly sample the one-day time pattern from January and apply it to the monthly total for January from the global climate model). For example, ten different one-day temperature and precipitation time-series were generated for each global climate model output (like a Monte Carlo analysis). Nine different climate models were chosen and generated ten "weather generations" for each (ninety total simulations) model. The simulations were divided into four periods, a historical period from 1950 - 2000 and three future periods, 2011-2040, 2041-2070, and 2071-2100. The computed output from the HEC-ResSim model, at Fargo, North Dakota, was used to determine the annual peak flows and frequency curves were computed using model output. One goal was to determine whether the global climate models are predicting larger flows in the Red River watershed.

\subsection{Dam Safety Studies (Bluestone and Success)}

CEIWR-RMC (Risk Management Center) contacted CEIWR-HEC about application of the stochastic modeling capabilities within HEC-WAT to dam safety studies. HEC-WAT/FRA is being used on multiple studies to extend flow and reservoir stage frequency curves out to the Probable Maximum Flood (PMF) event. Additional capabilities will be developed in phases and includes new capabilities to the Hydrologic Sampler, and new Monte Carlo simulation capabilities being added to HEC-HMS for sampling initial snowmelt parameters and correlation between model parameters. HEC-WAT was used for the Bluestone Dam [11] Hydrologic Loading Assessment that was conducted by CEIWR-HEC, CEIWR-RMC, and the Huntington, West Virginia office of USACE (CELRH). The HEC-WAT Monte Carlo simulation was used to sample the flood season, flood magnitude, hydrograph shape, and initial reservoir condition while HEC-ResSim was used to route the flood hydrographs and simulate operations of the dam. Peak reservoir stages were extracted from model results and used to define the hydrologic loading curve.

In another example, an HEC-WAT analysis was applied to a watershed on the eastern slope of the Sierra Nevada Mountains in central California. The Monte Carlo tools in HEC-HMS and HEC-ResSim within the HEC-WAT framework were used to estimate a reservoir stage frequency curve for the Success Dam watershed [12]. A reservoir pool stage frequency curve, including an estimate of the 90percent confidence limits, was required from the hydrologic analysis for a dam safety study. The pool stage frequency curve must be extrapolated to peak elevations not yet experienced in the reservoir, such as the peak pool elevation generated by the PMF event. The pool stage frequency curve was used, along with hydraulic and consequence modeling, to estimate the damage and life loss for various possible failure modes of a dam. Extending the reservoir pool stage frequency curve out to the peak elevations not yet experienced by the reservoir is challenging due to the assumptions and uncertainties of routing synthetic events, including the PMF. The HEC-WAT/FRA compute option was used to sample meteorologic forcings, initial models states and hydrologic modeling parameters to help define the reservoir stage frequency curve. Output from the HEC-WAT/FRA simulation included the sampled basin average precipitation (from the Hydrologic Sampler), maximum flow at user defined points (from HECHMS), and maximum flow/stage for reservoir elements (from HEC-ResSim). Output from one realization can be used to create a frequency curve by ranking reservoir peak stage values from largest to smallest and assigning an exceedance probability based upon the rank and number of events (the largest value will have an exceedance probability of $1 / n+1)$. For example, a realization of 10,000 events can be used to create a frequency curve that extends to the 0.01 percent exceedance probability. An FRA simulation that is configured with 100 realizations will have 100 estimates of the frequency curve which can be used to define the best estimate frequency curve and the confidence limits. The HEC-WAT/FRA simulation is an improvement from the common assumption made when estimating probabilities of flow or stage, that the 1-percent precipitation generates the 1-percent flow which in turn generates the 1-percent reservoir stage. The HEC-WAT/FRA simulation can sample many scenarios, like one where the 50-percent precipitation coupled with a high starting storage results in a 0.1-percent reservoir elevation. The Hydrologic Sampler within HEC-WAT was used to sample both precipitation and temperature, which was used as boundary conditions for an HEC-HMS model of the watershed. The HECHMS model sampled initial conditions (initial soil moisture) and model parameters, like infiltration rates, before computing inflow into the Success Dam reservoir. Finally, the HEC-ResSim model sampled initial storage within the reservoir and then routed the flow computed by HEC-HMS while maintaining downstream flow targets. The peak reservoir stage was extracted from the event simulations to build the reservoir stage frequency curve from 100,000 event realizations. 


\subsection{Levee Safety Studies (St. Paul and Natomas)}

The St. Paul Levee Pilot Study was performed for the USACE Levee Safety Team using HEC-WAT. The Levee Safety Team was investigating what tool to use for their national levee safety evaluation efforts. HEC-WAT with the Flood Risk Analysis (FRA) compute option was compared to other tools. In this instance of HEC-WAT, the hydrologic sampler, the fragility curve sampler, HEC-RAS and HEC-FIA were used. A flow frequency curve generated from gage data on the Mississippi River was sampled along with typical hydrograph shapes by the hydrologic sampler within HEC-WAT to provide a hydrograph for each event. The fragility curve was then sampled and that information was compared to the estimated water surface elevations generated from HEC-RAS for each event. Manning's roughness values were sampled for each HEC-RAS run and then for the events where the estimated stage exceeded the sampled value from the fragility curve, the levee was made to fail and water was then allowed to move into the interior. The flows that moved into the interior area were used to generate damage and life loss estimates. Following this study, the USACE Levee Safety Team agreed that HEC-WAT had promise as a tool to be used for their National program.

The USACE Levee Safety Team is currently performing another levee safety study where they are evaluating the use of HEC-WAT for their levee safety program. However, in this case, they are using the Natomas area of the city of Sacramento, California for their evaluation. The objective of this study is to determine the change in risk over time if a levee improvement is performed. The levee improvement will promote development which will lead to a population increase in the Natomas area. The HECWAT analyses will determine life loss in the area based on several alternatives being considered. Also, the analysis will take a look at the questions - How do levee degradation, dam re-operation, and climate change impact risk over time? Unlike the simple St. Paul example noted above, the Natomas basin is much more complex. The area is completely surrounded by levees and can be exposed to high water from multiple sources. The composition of the levees themselves are variable and the possible failure mechanisms are multiple. In this case, the HEC-WAT runs include the hydrologic and fragility curve sampler but this time, there are multiple sources of flow and multiple possible failure locations. HECRAS and HEC-FIA are also being used but again, the modeling is far more complicated than the St. Paul example. At the time of the writing of this paper, study evaluation was still being conducted.

\section{Conclusion}

To help study and manage the nation's water resources in a holistic and comprehensive approach while implementing system and risk concepts,
USACE developed and has now implemented HECWAT. The application of HEC-WAT with the FRA compute option (HEC-WAT/FRA) on a variety of studies clearly demonstrates that it is a tool that begins to meet USACE requirements and address the needs of complex and sophisticated studies as recommended by the National Research Council.

Some of the technical challenges that still need to be overcome include how to incorporate life cycle modeling that addresses rehabilitation, repair and flood recovery; consequence evaluation; uncertainty analysis trade-offs between detailed modeling and important sources of uncertainty; risk communication; modeling multiple failure modes; planning transformation; and the future of planning analysis in USACE. In spite of these technical challenges, HECWAT/FRA is being used on a variety of studies to help answer a variety of difficult questions. In addition, because of its unique set of capabilities, International interest in HEC-WAT is also starting to rise.

The HEC-WAT development team, along with other software development teams at CEIWR-HEC, which include HEC-RAS, HEC-FIA, HEC-ResSim, and HEC-HMS, are continuing to look for additional innovations to improve model performance during Monte Carlo simulations. Innovations include decreasing simulation times and providing better options for generating and storing model output. These improvements are not as necessary for typical studies in which models are simulated only a few times, but the improvements become much more critical when simulating 50,000 or more events for Monte Carlo type simulations. A version of HECWAT (Version 1.0) with the FRA compute option will be available in late 2016 and will include the capabilities discussed in this paper.

\section{References}

1. U.S. Army Corps of Engineers (2000). Planning Guidance Notebook, ER 1105-2-100.

2. U.S. Army Corps of Engineers (2006). Risk Analysis for Flood Damage Reduction Studies, ER 1105-2-101.

3. Hydrologic Engineering Center (2014). HEC-FDA Flood Damage Reduction Analysis, User's Manual, 378 pages.

4. National Research Council (2000). Risk Analysis and Uncertainty in Flood Damage Reduction Studies, National Academy Press.

5. Dunn, C. and Baker, P. (2010). A Watershed Modeling Tool, HEC-WAT. ASCE Watershed Management 2010: Innovations in Watershed Management under Land Use and Climate Change, pp. 1101-1112.

6. Dunn, C., Baker, P., and Fleming, M. (2014). Flood Risk Management with HEC-WAT and the FRA Compute Option. ICFM6, $6^{\text {th }}$ International Conference on Flood Management, 2014, Sao Paulo, Brazil. 
7. Dunn, C., and Baker P. (2012). A Nationwide Comprehensive Water Management System: Can We Get There and Why Should We Care. 16th SANCIAHS National Hydrology Symposium 2012, Pretoria, South Africa

8. Hydrologic Engineering Center (2008). Model Design for Flood Damage and Risk Analysis of Complex Riverine Systems, 47 pages.

9. U.S. Army Corps of Engineers (1996). Risk-Based Analysis for Flood Damage Reduction Studies, EM 1110-2-1619.

10. United States Entity, Columbia River Treaty (2013). U.S. Entity Regional Recommendation for the Future of the Columbia River Treaty after 2024

11. Hydrologic Engineering Center (2015). Development of the Hydrologic Loading Curve for Bluestone Dam, Project Report no. 98, 65 pages.

12. Fleming, M and Duren, A. (2014). Stochastic Hydrologic Simulation for Extension of Reservoir Pool Stage Frequency Curves. ASCE World Environmental and Water Resources Congress 2015: Floods, Droughts, and Ecosystems, pp. 2648-2657. 